\title{
Neural Correlates of Feature Selective Memory and Pop-Out in Extrastriate Area V4
}

\author{
Brad C. Motter \\ VA Medical Center and Departments of Physiology and Neurosurgery, SUNY Health Science Center, Syracuse, New York \\ 13210
}

Neural activity in area V4 was examined to assess (1) whether the effects of attentive selection for stimulus features could be based on the memory of the feature, (2) whether dynamically changing the feature selection would cause activity associated with the newly selected stimuli to pop out, and (3) whether intrusion of more than one stimulus into the receptive field would disrupt the feature-selective activity. Rhesus monkeys were trained on several variations of a conditional orientation discrimination task. A differential activation of area V4 neurons was observed in the conditional discrimination task based on the presence of a match or a nonmatch between the conditional cue (a particular color or luminance) and the color or luminance of the receptive field stimulus. The differential activation was unchanged when the cue was removed and the animal had to remember its color (or luminance) to perform the task. When the cued feature was switched from one alternative to another in the middle of a trial the differential activation of neurons reversed over the course of 150-300 msec. If the stimulus in the receptive field contained the newly selected feature, V4 neurons became activated without a concomitant change in the stimulus in classical receptive field. Across the topographic map of V4 the activity associated with the newly selected stimuli popped out, whereas the activity of deselected stimuli faded to the background levels of other background objects. Evidence of a suppressive input from stimuli outside the classical receptive field was clear in only 3 of 24 neurons examined. Intrusion into the classical receptive field by a second stimulus resulted in a diminished difference between matching and nonmatching conditions. These physiological data suggest a major role for attentional control in the parallel processing of simple feature-selective differences.

[Key words: attention, preattentive vision, feature selection, pop-out, visual memory, area V4]

Previous physiological studies have demonstrated that focal attentive tasks are effective in emphasizing the visual processing of relevant stimulus information within extrastriate area V4 (Moran and Desimone, 1985; Haenny and Schiller, 1988; Haenny et al., 1988; Spitzer et al., 1988; Maunsell et al., 1991; Motter, 1993). The results reported in the preceding companion article

\footnotetext{
Received June 25, 1993; revised Sept. 13, 1993; accepted Sept. 29, 1993.

This work was supported by the VA Medical Research program.

Correspondence should be addressed to Brad C. Motter, Research Service 151, VA Medical Center, 800 Irving Avenue, Syracuse, NY 13210.

Copyright (C) 1994 Society for Neuroscience 0270-6474/94/142190-10\$05.00/0
}

(Motter, 1994) demonstrate a second more general selective process, occurring in parallel across the visual scene and based on the selection of a color or luminance stimulus feature. Under the conditions of those experiments the activity of neurons within the V4 population reflected a selection of a stimulus feature rather than a response to the presence of a particular physical color or luminance within the receptive field. The consequence of the feature-selective process is that on any given trial the majority of the highly activated V4 neurons preferentially represent those separate stimuli in the visual scene that correspond to potential targets as defined by their color or luminance. This parallel feature-attentive process may be the physiological counterpart of an attentive highlighting of locations in the visual scene to which further focal attentive processing can be directed.

The studies reported here had three primary goals. The first goal was to determine whether the feature-selective process could be maintained in the absence of the visible cue. Since other studies had shown that V4 neuronal activity could be modulated within a delayed match-to-sample paradigm, even by cues from nonvisual sources (Haenny et al., 1988; Maunsell et al., 1991), the design chosen examined whether the feature-selective process could be maintained by the memory of the cue condition. The second goal was to determine whether the selection process could be altered once it was established for a particular behavioral trial. Shifts in the selection process provide a measure of the time course over which parallel processes can shift from one subset of stimuli to another. The term "pop-out" is used in this article to describe the sudden emergence throughout the visual field of the neural representation of a set of stimuli sharing a particular feature. The third goal was to investigate the influence of stimuli in the suppressive surrounds of V4 neurons upon the discharge response to stimuli presented in the classical receptive fields. Previous reports have found that focal attentive mechanisms govern interactions within the classic receptive field (Moran and Desimone, 1985). This study was to examine whether the parallel mechanisms of the feature-selective processes were specifically excluded from the classic receptive field or the suppressive surrounds.

\section{Materials and Methods}

\section{Behavioral paradigms}

This report describes data from two of the three behaviorally trained Macaca mulatta monkeys that served as subjects in the preceding article. These monkeys were first trained on the conditional discrimination (CD) task described below and in the preceding article (Motter, 1994). The two monkeys were then additionally trained on two different variations of the standard task.

Standard conditional discrimination paradigm. Each of these monkeys was trained on a two-stage conditional orientation discrimination 
(CD) task that required the discrimination of the orientation tilt of a bar stimulus once it was selected from a pair of stimuli based on its color or luminance. In all tasks the subjects first visually fixated a small circular fixation spot whose color or luminance provided the conditional cue for the trial. After the animals established fixation of the spot, an array of four or six stimulus bars composed of combinations of two orientations and two colors or luminances was presented. In order to study spatial interaction effects between stimuli and receptive field regions, some series of studies employed additional arrays of 8,12 , or 16 stimuli. In the standard task thesc conditions persisted throughout the trial until the end, when the array was reduced to just two stimuli, only one of which matched the fixation cue color or luminance. At this point the subjects made an orientation discrimination of the matching color (or luminance) stimulus by pushing either a righthand button for rightward tilts of the bar stimulus or a lefthand button for leftward tilts.

Memory paradigm. The first variation of the conditional discrimination paradigm was designed to require the monkey to make the final selection of the appropriate bar stimulus based on a memory of the color or luminance of the conditional cue rather than on any possible comparisons of stimuli in the visual field. This was accomplished by simply turning off the fixation spot part way through the trial (see Fig. 1 , left). The monkeys were required to maintain fixation at the same point on the screen and make the conditional choice at the end of the trial based on the color of the fixation spot prior to its disappearance. The fixation spot was turned off either 750 or $1000 \mathrm{msec}$ after the onset of the array. The array remained on for an additional 1000-1800 msec after the fixation spot was turned off before being reduced to the final two stimuli upon which the conditional choice and discrimination was made.

Cue switch paradigm. A second variation of the standard conditional discrimination paradigm was designed to alter the conditional cue color or luminance partway through the trial and thereby require the final conditional selection process to be based on information received after the array was present in the visual field. This was accomplished by switching the color or luminance of the fixation spot from one of the two alternatives to the other (see Fig. 1, right). The timing parameters were similar to those used in the memory paradigm.

Simple discrimination paradigm. Prior to studying each neuron on the conditional task the neuron's receptive field was located and mapped and the response preferences for stimulus size, color, and orientation were determined using a simpler discrimination paradigm designed to approximate the standard fixation paradigm commonly used in visual studies (Wurtz, 1969). This paradigm required the monkey to ignore peripheral stimuli while fixating a central spot until a small bar was flashed directly over the fixation spot. The monkey then made an orientation discrimination based on the small flashed bar. In addition to the standard single test and stimulus mapping of receptive fields, two or three simultaneously presented stimuli were used to assess the suppressive zones surrounding area V4 neurons.

\section{Stimulus presentation and data collection}

Details of stimulus generation and data collection were the same as those described in the preceding companion article (Motter, 1994). Briefly, stimuli were generated on a graphics display monitor located $60 \mathrm{~cm}$ in front of the monkey. Stimulus timing and eye position sampling were locked to the $60 \mathrm{~Hz}$ frame rate of the display system. Each series of stimulus conditions was governed by a quasirandom permutation series so that trial-to-trial stimulus selection could not be predicted. The stimuli consisted of rectangular bars of various sizes, oricntations, and colors presented against a background gray of $7.5 \mathrm{~cd} / \mathrm{m}^{2}$. The fixation target was a small filled circle $\left(0.2^{\circ}\right)$. Stimulus arrays usually contained either four or six bar stimuli equally spaced along an imaginary circle centered on the fixation spot. The array was placed so that one bar stimulus was centered in the receptive field of the neuron being studied. A standard set of colors $\left(34 \mathrm{~cd} / \mathrm{m}^{2}\right)$ and gray scale values was used.

Each animal viewed the display monitor binocularly through an infrared reflecting mirror placed directly in front of its eyes. Eye position of the left eye was recorded with an infrared corneal reflection oculometer (Motter and Poggio, 1984). Steady fixation of the central fixation spot without eye blinks or eye movements to peripheral sites was required during the entire stimulus sequence. The monkeys worked daily for about 1200-1400 trials. Correct responses were rewarded after each trial. Animals were maintained on a controllcd liquid intake schedule and received their baseline amount each day either in the course of

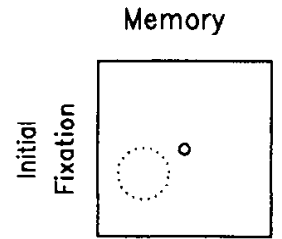

Cue Switch
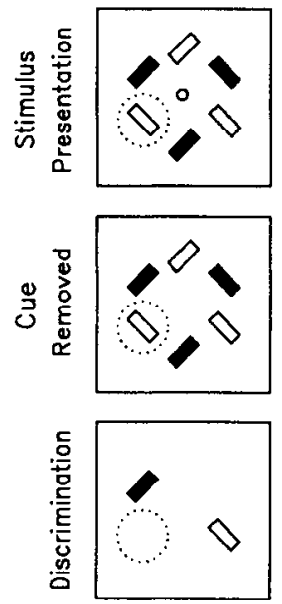
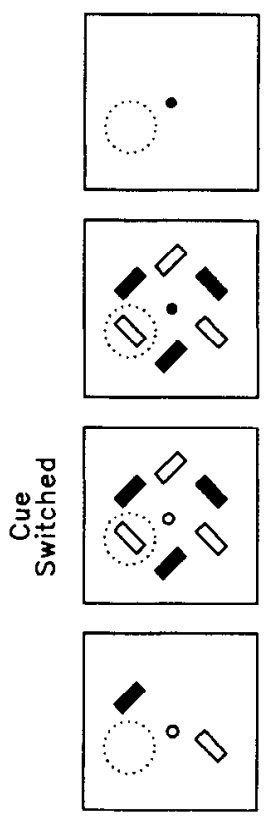

Figure 1. Behavioral paradigms. Each frame represents a ficld of vicw with the fixation spot in the center and the neuron's receptive field indicated by the dotted circle. For either task the subject initially fixates the small fixation spot and is then presented with an array of stimuli. In the memory paradigm the fixation spot disappears 750 or $1000 \mathrm{msec}$ after the array onset. The subject must remember the color of the fixation spot so that when the array is reduced to an unambiguous choice of two stimuli the subject can discriminate and report the orientation of the bar stimulus whose color matched that of the fixation spot. The CS paradigm is similar except that instead of disappearing, the fixation spot (the color cue) changes color and the subject must use this new color to choose the correct har stimulus and discriminate its orientation. $M$ and NM conditions for both paradigms are distinguished by whether or not a color or luminance match occurs between the fixation spot and the stimulus in the receptive field. The figure is not scaled proportionately.

working on the tasks or supplemented afterwards. Animals received weekly holidays from the schedule.

\section{Procedure}

A subset of the neurons selected for study in the previous report were also tested in the paradigms reported here. These single neurons responded well to flashed stimulus bars of various colors and luminances that were about $2.5^{\circ} \times 0.5^{\circ}$ in size. The orientation, color, and luminance preferences, and receptive field location were mapped using a series of flashed stimuli under computer control. Two orientations and two colors/luminances were chosen from these results. The preferred orientation and best color stimulus were then used to redefine the edge of the receptive field nearest the fixation point and to cxaminc the borders of the receptive field along an arc equidistant from the fixation target and passing through the receptive field center. The spacing of stimuli in the array of the conditional discrimination paradigm was then chosen so that only one stimulus would be present in the receptive field on any trial; exceptions to this statement are discussed in the Results. After all stimulus parameters were defined using the simpler discrimination paradigm, the monkey was switched to the standard conditional discrimination paradigm. Following the standard paradigm, neurons were studied while the monkey worked on either the memory or cue switch (CS) paradigms.

Behavioral training, neurophysiological recording, and verification of recording sites were the same as reported in the companion article (Motter, 1994) and a previous report (Motter, 1993). All experimental protocols were approved by the Animal Care and Usc Committees at the VA Medical Center and SUNY-HSC. 
Figure 2. Responses of a V4 neuron to the primary stimulus in both the standard $C D$ paradigm and the memory paradigm. Raster displays of discharge activity show the difference between match $(M)$ and nonmatch $(N M)$ conditions during trials for both paradigms. Stimulus array onset is marked by vertical bars on the left. In the memory paradigm removal of the fixation spot $750 \mathrm{msec}$ after the array onset does not alter the differential response for either $\mathrm{M}$ condition. For a few neurons including this one, responses in the memory paradigm were more strongly differentiated than those in the CD paradigm.
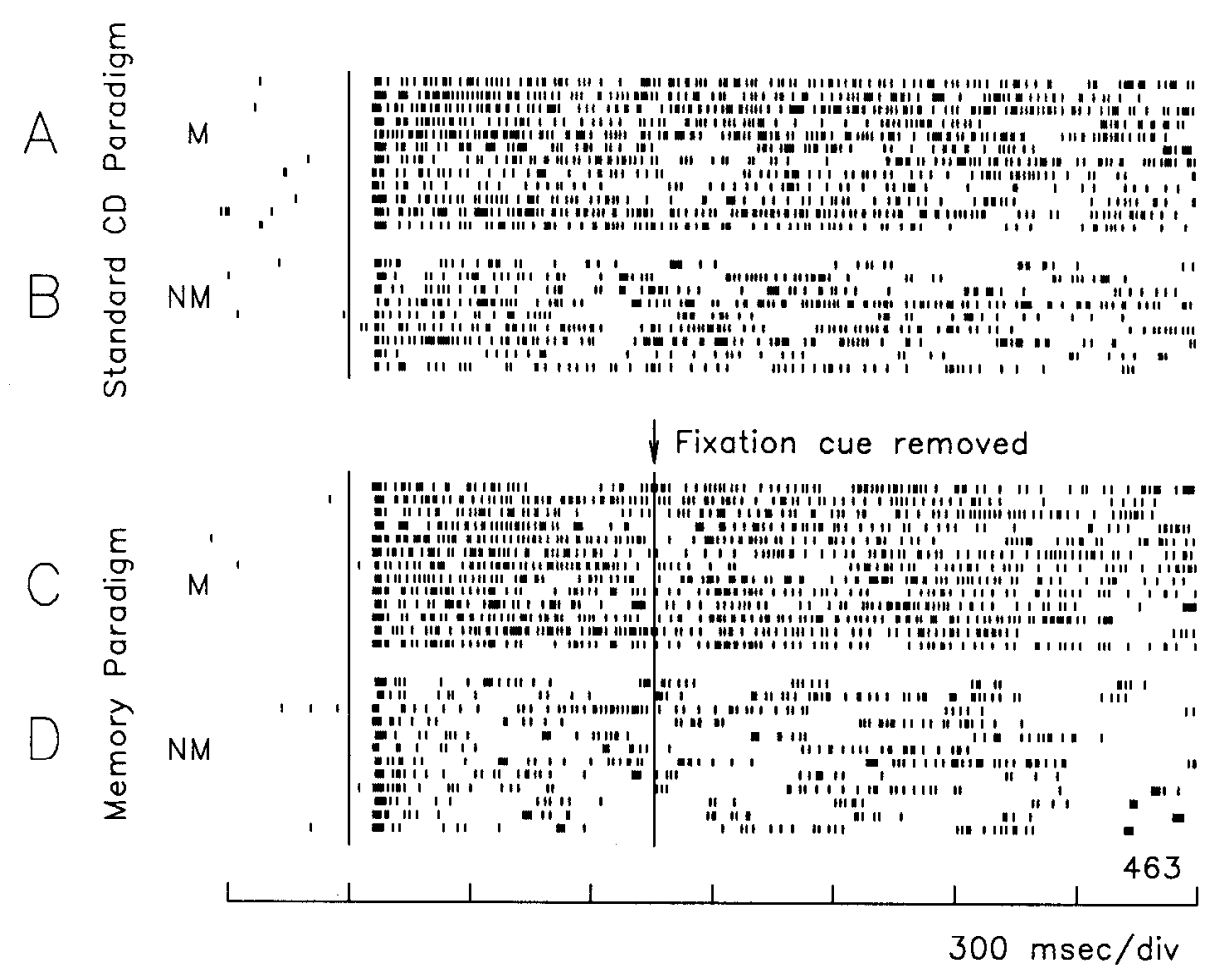

\section{Results}

Subsets of the $109 \mathrm{~V} 4$ neurons described in the previous article were further studied in the several variations of the $C D$ paradigm presented in this article. All of these neurons were recorded in the exposed portion of the prelunate gyrus within the area defined as area V4 by Gattass et al. (1988). The receptive fields and basic stimulus response preferences were determined using stimuli presented one at a time in the simple discrimination paradigm. Receptive field centers were located within the range from $3^{\circ}$ to $6^{\circ}$ of the foveal center.

\section{Differential activation based on a memory of the cue}

The memory paradigm provided an assessment of whether the maintained differential responses observed with the CD paradigm could be sustained by a memory of the appropriate cuc color/luminance. A total of $32 \mathrm{~V} 4$ neurons were studied using the memory paradigm. Each of these neurons had receptive fields centered $3-6^{\circ}$ from the fixation spot that did not extend to include the fixation point within the receptive field. This condition was defined by locating the position of the edge of the receptive field between the fixation spot and the center of the receptive field using standard single stimulus mapping procedures. Each neuron was first studied in the standard CD paradigm. Figure 2 illustrates the results for one V4 neuron. The rasters in the upper part of the figure illustrate the responses under the matching (M) and nonmatching (NM) conditions of the standard $C D$ paradigm. Within each paradigm all stimulus conditions were randomly interleaved trial by trial. Only the responses to the primary stimulus (color, red; orientation, $45^{\circ}$ ) are shown in this figure; responses to the other stimuli are not shown. The trials on which the receptive field stimulus matched the conditional cue are shown in Figure $2 A$, and trials for the NM condition in the standard CD paradigm as shown in Figure $2 B$. The trials are synchronized to the onset of the array indi- cated by the vertical bar on the left. The response of the neuron in the NM condition of Figure $2 B$ is reduced compared to the $M$ condition. The response of this same neuron during the memory paradigm is shown in the lower part of Figure 2. Again, the responses are shown for only the primary stimulus, as above, and the trials are sorted here into $\mathrm{M}$ and NM conditions. The second vertical bar, $750 \mathrm{msec}$ after the onset of the array, denotes the time that the fixation spot was removed. No change is noted in the neural activity. The activity continues to show a differential maintained response related to the $\mathrm{M}$ and NM conditions. For some of the neurons (see Fig. 2) the responses in the memory paradigm were more strongly differentiated than the responses in the standard CD task.

Average response rates in a one second interval after the removal of the fixation spot were calculated for each condition in the memory paradigm. Average rates were obtained from a similar interval in the standard CD paradigm for the same neurons and the data were analyzed with a two-factor ANOVA with repeated measures. The matching condition effect was significant $(p<0.001)$. Differences between specific groups were examined with pairwise multiple comparisons. Significant differences for the $M$ and NM conditions were present for both paradigms. The effects were identified in individual neurons by $t$ test comparisons. Twenty-three of the 32 neurons tested were significantly different in the standard CD task and all 23 of these were also significantly different in the memory paradigm $(p<$ $0.05, t$ test). An additional four neurons not different in the standard task were significantly different in the memory paradigm, making a total of 27 out of 32 neurons with clear differences between $M$ and NM conditions in the memory paradigm. There was also a small but significant $(P<0.01)$ difference in the overall ANOVA between the standard condition and the memory paradigms that could be attributed to slightly higher discharge rates in the standard $M$ condition. Figure 3 summarizes the comparison between responses in the standard para- 


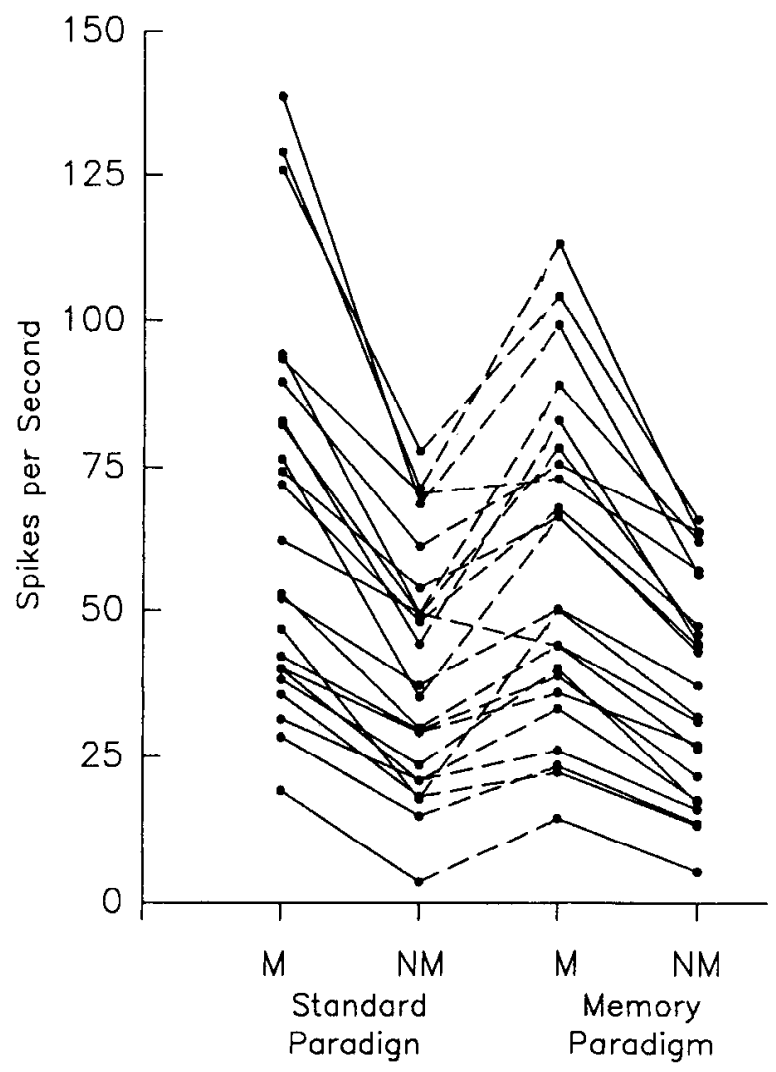

Figure 3. Mean discharge rates for match $(M)$ and nonmatch $(N M)$ conditions for $23 \mathrm{~V} 4$ neurons during both the standard CD paradigm and the memory paradigm. The average firing rate for each condition is joined by a line for each neuron. Discharge rates in $M$ conditions exceeded those in NM conditions for both paradigms. digm and the memory paradigm in the set of 23 neurons showing clear differences between $M$ and NM conditions in both paradigms. The average firing rate in each condition is plotted and joined by a line for individual neurons. The NM condition is always attenuated relative to the $\mathrm{M}$ condition.

These results indicate that a memory of the cue is sufficient to sustain the differential neural activity. They also offer a further demonstration that the observed differential responses are not due to simple stimulus-stimulus interactions between the fixation spot and the bar stimuli in the array. In contrast to this result, the presence of a stimulus in the receptive field was an absolute requirement. If the stimulus was removed or if an crrant eye movement moved the receptive field away from the stimulus the neural activity ceased abruptly. Therefore, the sustained response is not a mnemonically driven but a stimulus driven activity that can be apparently gated or modulated by mnemonic processes.

\section{Effects of switching the selected stimulus feature: pop-out}

The memory paradigm demonstrated that the cue information did not have to be present during the trial to sustain the differentiated activity. It was therefore necessary to show whether the sensitivity to $\mathrm{M}$ and $\mathrm{NM}$ conditions could be altered during the behavioral trial if the cuing conditions were changed. Such conditions represent a more natural situation where stimuli already present in the visual field either acquire or lose significance relative to the task at hand.

Neurons were first studied in the standard CD paradigm. A second scrics of trials using the CS paradigm was then begun if a differential response in the standard CD paradigm was evident. A total of $40 \mathrm{~V} 4$ neurons were studied using the CS paradigm. An example of the response of a V4 neuron in both of these paradigms is shown in Figure 4 . The receptive field stimulus is

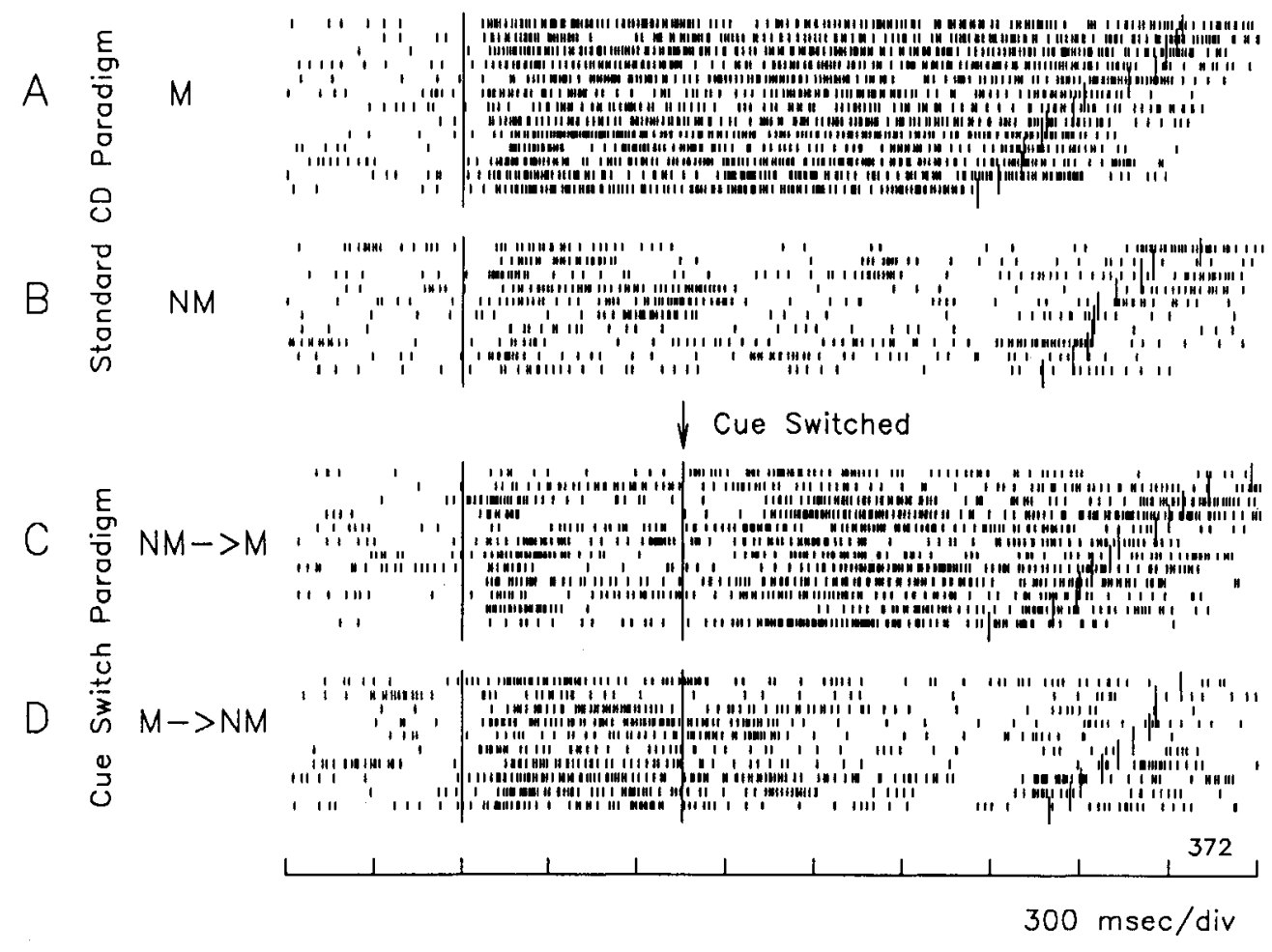

Figure 4. Responses of a V4 neuron to the primary stimulus in both the standard CD paradigm and the CS paradigm. Raster displays of discharge activity show the difference between match $(M)$ and nonmatch $(N M)$ conditions during trials for both paradigms. Trials are sorted by condition and by trial duration. Short staggered vertical lines at the right are the times of behavioral key release at the end of the trial. Stimulus array onset is marked by vertical bars on the left. In the CS paradigm the $M$ and NM conditions reverse when the cue switches at 750 msec after array onset. The activity of the neuron also switches after a delay of about $200 \mathrm{msec}$. The response change occurs without any change in the stimulus in the classical receptive field. 


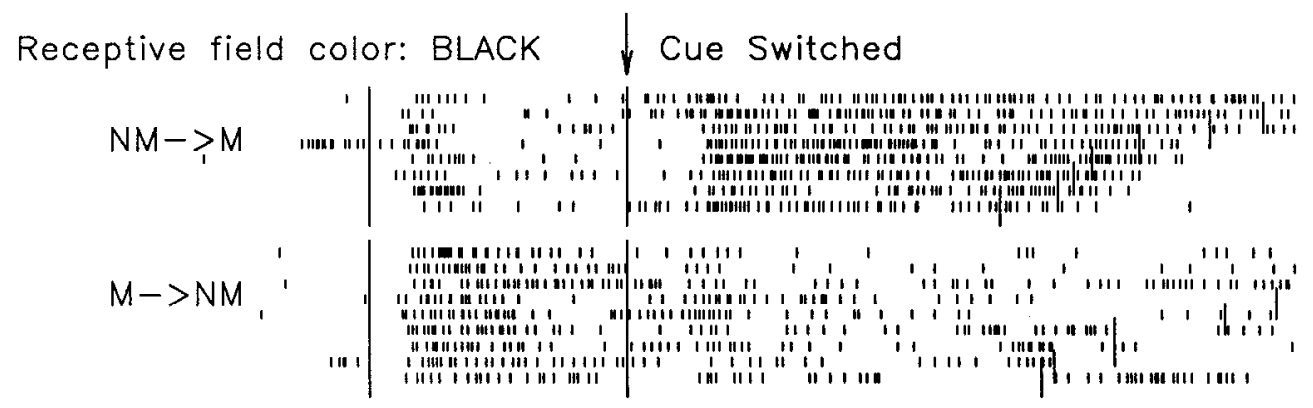

Receptive field color: BLUE

Figure 5. Responses of a V4 neuron to both primary and secondary stimuli in the CS paradigm. For either stimulus the response pops out when an NM condition becomes an $\mathrm{M}$ condition (NM $\rightarrow M$ ). Likewise the response fades away when the change is from $\mathrm{M}$ to NM $(M$ $\rightarrow N M)$. Presentation format is similar to Figure 4.

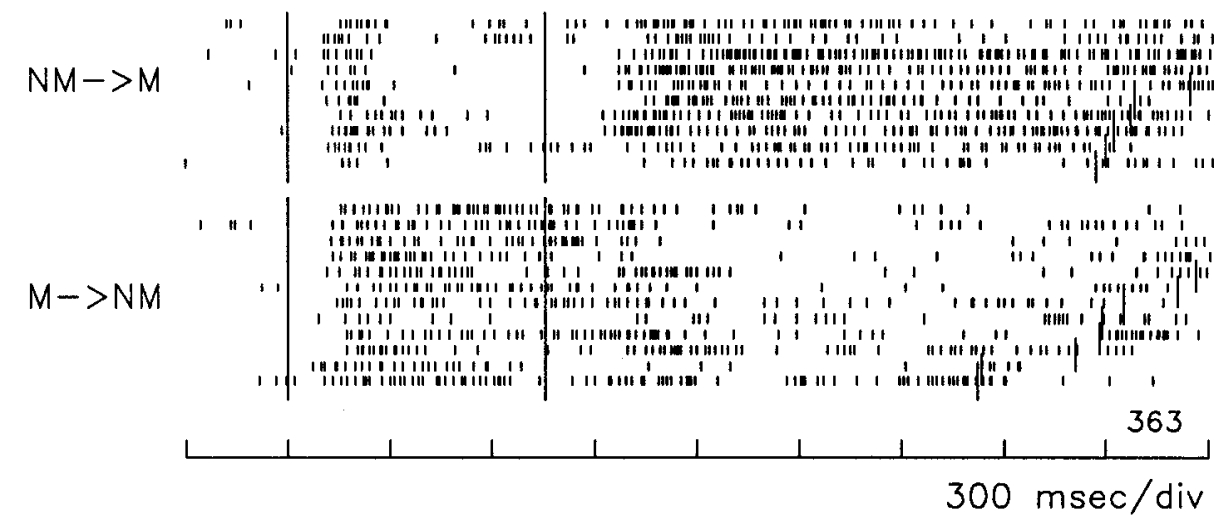

the primary stimulus (color, blue; orientation, $60^{\circ}$ ) in all cases in Figure 4. The upper pair of rasters illustrates the response of the neuron during the $\mathrm{M}$ (Fig. $4 A$ ) and NM (Fig. $4 B$ ) conditions of the standard $C D$ paradigm. The response in the NM condition returns nearly completely to the low level of spontaneous background activity exhibited by this neuron. The lower pair of rasters illustrates the response of the neuron during the CS paradigm. The upper raster of the lower pair (Fig. $4 C$ ) illustrates an initially NM condition with the low level of activity seen in Figure $4 B$. Then, $750 \mathrm{msec}$ after the onset of the stimulus array, the fixation spot color is switched (from white to blue) and the response of the neuron also switches after a delay to the activation pattern of response similar to the $M$ condition in the standard paradigm shown in Figure $4 A$. Figure $4 D$ shows the opposite direction of change from an initially $M$ condition to an NM condition and the accompanying switch in neural activity from an activation to an attenuated response.

Figure 5 shows the activity of a second V4 neuron during the CS paradigm neuron. This neuron was strongly selective for blue and black stimuli when tested with $200 \mathrm{msec}$ flashed stimuli in the simple discrimination paradigm. Under those conditions the contrast index $(A-B) /(A+B)$ for blue versus green was 0.95 ; for blue versus red, 0.86 ; for black versus white, 0.58 ; and for black versus blue, 0.06 . When these two "colors" were used in the CS task the neuron's activity could be toggled on or off for either color by providing the appropriate conditional cue. Thus, changes in the cue cause a dramatic shift in the neural activity in the absence of any changes in the receptive field stimulus. In a topographic map of the population activity, neurons that represent the stimuli that become selected pop out, whereas neurons representing stimuli that become deselected fade away.

As a population, the 40 neurons studied in the CS paradigm attained significant reversals of their activity for either direction of cue switching. Average spike rates were calculated in a 750 msec interval before the cue switch and a 750 msec interval beginning $250 \mathrm{msec}$ after the cue switch. Figure 64 summarizes the activity in these periods. Data points for the change from $M$ to NM conditions are shown as open circles and for the change from NM to $\mathrm{M}$ conditions are shown as solid circles. The data points separate into two distinct groups depending upon whether there was a match to the conditional cue before (open) or after (solid) the cue switch. The data shown in Figure $6 \mathrm{~A}$ represent the shift in ncural activity that occurs, but because activity was measured at different times in the trial and because the overall level of activity changes during the trial, the differences might be slightly overstated. Measures of activity were therefore made between the periods after the cue switch and comparable periods in the standard CD task. Thus, comparisons were made between activity levels that would have been present if the cue had not switched (CD paradigm) and activity levels after the cue actually did switch (CS paradigm). Two sets of contrast indexes based on the paradigms $(\mathrm{CD}-\mathrm{CS}) /(\mathrm{CD}+\mathrm{CS})$ were calculated, one for the $\mathrm{M}$ to NM change and one for the opposite change. Figure $6 B$ shows the distribution of indexes for the $\mathrm{M}$ to NM change. The activity of all but two neurons is depressed relative to what it would have been at the same time in the trial if the cue had not switched. Figure $6 C$ shows the distribution for the NM to $M$ change. The activity of all but two neurons is elevated relative to what it would have been at the same time in the trial if the cue had not switched. The neurons that failed to reach a clear difference after the cue switch did so mostly because they failed to change their firing substantially after a cue switch. Three neurons seemed to be directional, changing for one switch direction but not for the reverse.

\section{Time course of feature selection}

The CS paradigm also provided an assessment of the time course of the selection process within area V4 during a change from one stimulus feature to another. Because the visual field stimuli 

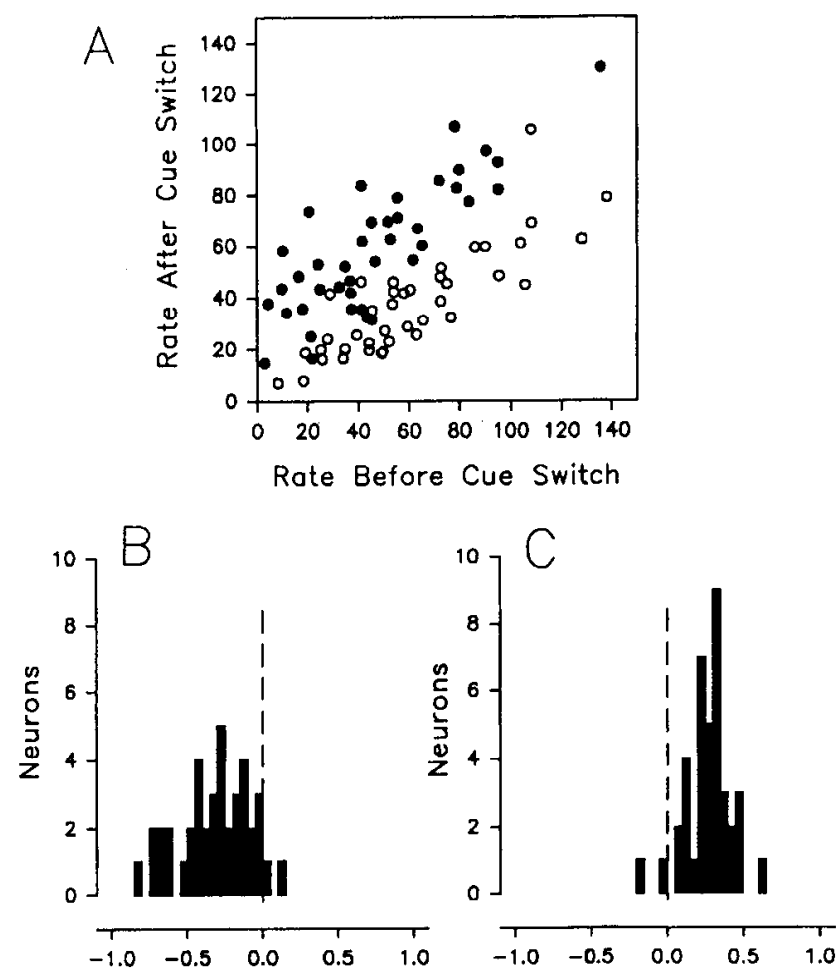

Non-Match <- Match

$$
\text { Non-Match } \rightarrow \text { Match }
$$

Figure 6. Summary of population activity in the CS paradigm. $A$, Average spike rates in intervals before and after the cue switch. Solid circles show activity for changes of NM to $\mathrm{M}$ conditions; open circles show the opposite $M$ to NM activity changes. $B$ and $C$, Distributions of the response contrast indexes for comparisons made between the standard CD paradigm and CS paradigm. In $B$, the comparison shows the decrease in activity for NM conditions after the switch. In $C$, the comparison shows the increase in activity for $M$ conditions after the switch.

were already present, including one in the receptive field of the neuron being studied, this assessment could be made without the concomitant activation associated with a stimulus onset. The changing patterns of response shown in Figures 4 and 5 occur without any change in the receptive field stimulus or stimulus array as a whole, and with only the cuing color change at the fixation spot. The time course of the change in neural activity potentially reflects the redirection of selective feature processing. To estimate this time course a population average of the activity was made. A subset of 30 neurons that showed the largest differences in the interval after the cue switch was used to provide a clearer picture of the temporal changes. The activity for each condition in each neuron was averaged in 10 msec intervals synchronized to the time of the cue switch. The response of each neuron was normalized with respect to its highest rate and the conditions were then averaged across the population of neurons. The resulting profiles of the time course of change in the CS paradigm are shown in Figure 7. The solid line represents a $50 \mathrm{msec}$ running average smooth of the averaged responses from the 30 neurons. The change in activity associated with switch from the $\mathrm{M}$ to $\mathrm{NM}(\mathrm{M} \rightarrow \mathrm{NM})$ conditions is somewhat delayed relative to the switch from the NM to $M$ $(\mathrm{NM} \rightarrow \mathrm{M})$ condition. The population activity change in the $\mathrm{NM} \rightarrow \mathrm{M}$ condition starts at about $150 \mathrm{msec}$, and for the $\mathrm{M} \rightarrow$ NM condition at about $300 \mathrm{msec}$, after the cue is switched. The

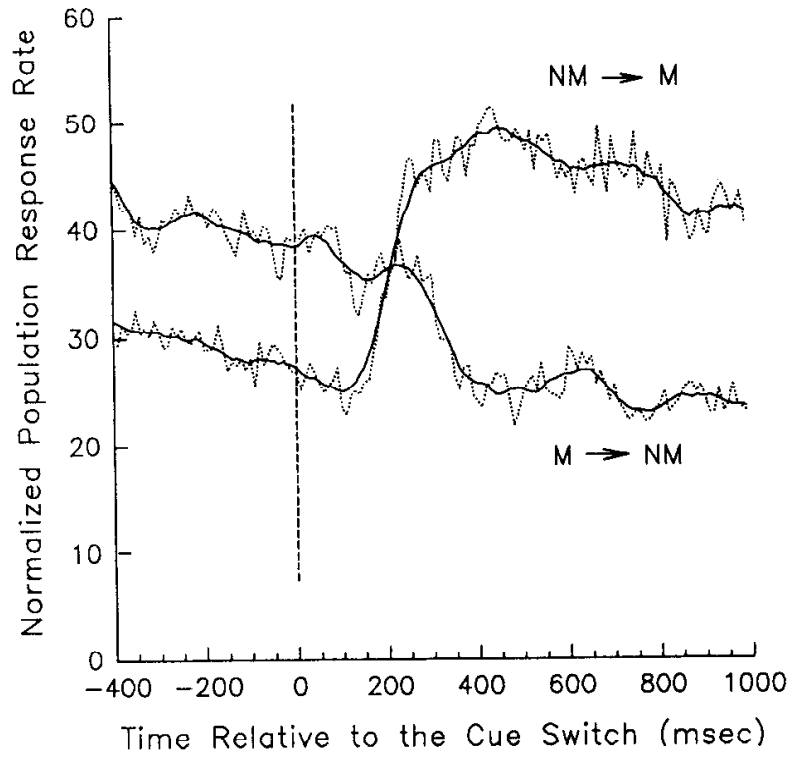

Figure 7. Time course of feature selection: normalized population histograms showing the activity changes accompanying switches from match to nonmatch $(M \rightarrow N M)$ and nonmatch to match $(N M \rightarrow M)$ conditions. Dashed vertical line marks the time of the cue switch. Histograms are based on $10 \mathrm{msec}$ bins. For each neuron the bin with the greatest activity across all conditions was set to 100 and the remaining bins normalized to that value. Data were then averaged across the 30 neurons. The solid line represents a $50 \mathrm{msec}$ running average smooth of the data.

staggered delays suggest an interval where both (or neither) feature is selected. For comparison, response differences after the initial presentation of the array, as shown in Figure 9 of the preceding article (Motter, 1994), begin at about 150-200 msec after the initial presentation of the array. These delays represent two to three times the standard visual latency observed in area $\mathrm{V} 4$ and indicate that a substantial processing interval is required for the setup of the feature-selective processes.

\section{Spatial interactions inside and outside the receptive field}

Under conditions of directed focal attention V4 neurons have been demonstrated to process information selectively within the receptive field (Moran and Desimone, 1985). The general design of the CD tasks used here was to place only one stimulus in the classical receptive field and the remaining stimuli well outside the boundaries of the receptive field. The receptive field was determined with a single stimulus presented in $200 \mathrm{msec}$ flashes while the monkey performed the task in the simple discrimination paradigm. To investigate the relationship of stimulus placement relative to the boundaries of the receptive field during the CD task, 24 neurons were studied using 8,12 , or 16 stimuli in the array in addition to the usual four or six stimuli. For most of these neurons attempts were also made to detect the inhibitory suppressive surround described for many V4 neurons (Desimone and Schein, 1987). These tests were made with the simple discrimination paradigm. Pairs of stimulus bars, one inside and one outside the classical receptive field, were flashed at locations along the imaginary arc defined by the eccentricity of the receptive field center from the fixation point. Evidence for the suppressive surround was difficult to cstablish (clcarly secn in only three neurons) using the bars that constituted the stimuli used in the arrays of this study.

In general, increasing the number of stimuli in the array in 


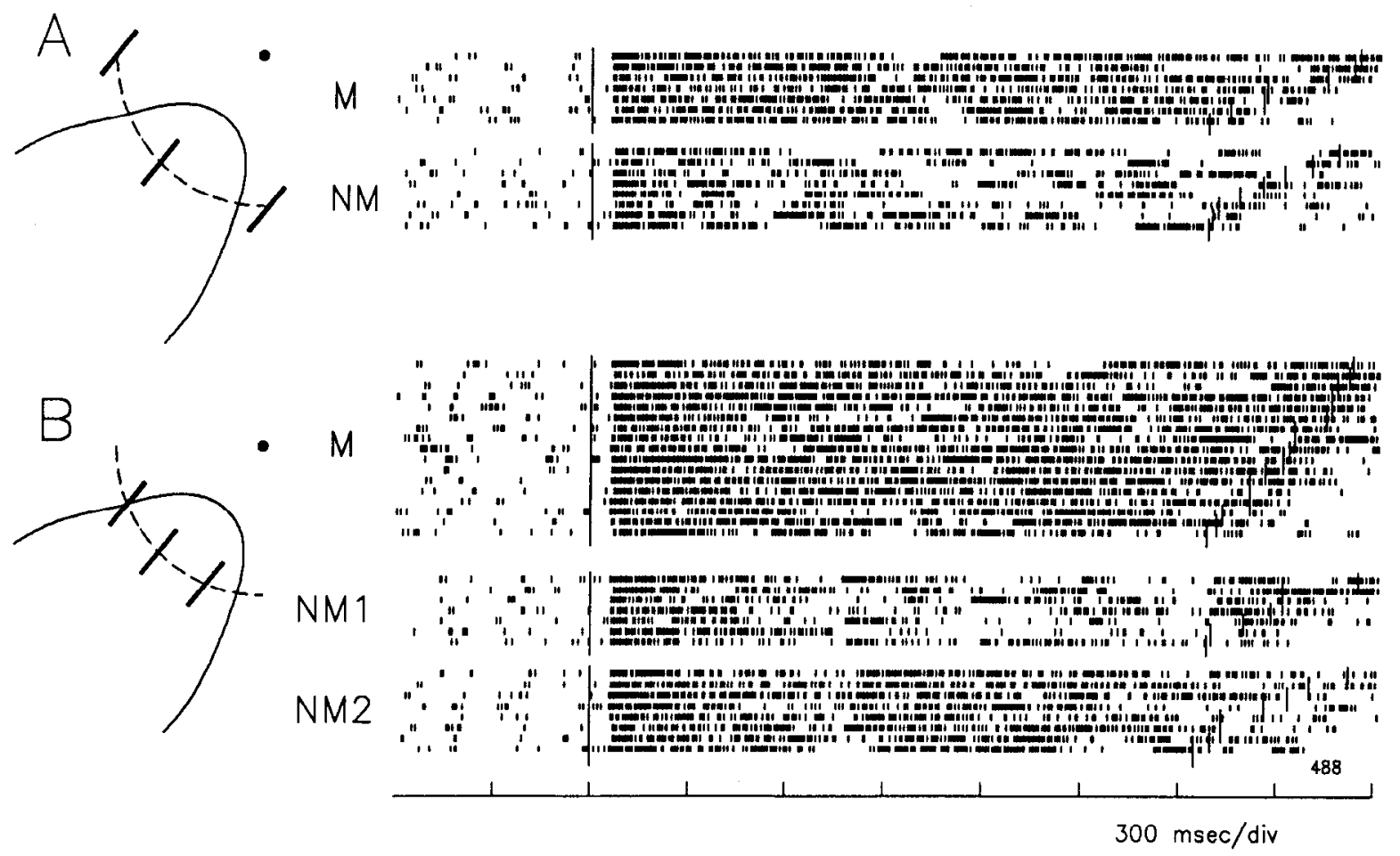

Figure 8. Entry of additional array stimuli into the receptive field: discharge responses of a V4 neuron to M and NM conditions in the standard CD paradigm for arrays containing eight stimuli $(A)$ and 16 stimuli $(B)$. Cartoon to the left depicts inner margin of the receptive field located to the lower left of the fixation spot. Bars represent the locations of the centered receptive field stimulus and its nearest neighbors in the array. In $A$, the differential responses to match $(M)$ and nonmatch $(N M)$ conditions are clear. In $B$, a second bar has clearly entered the receptive field. During the $M$ condition the response is unaltered and continues to indicate that an $M$ condition is present. During the NM condition two types of response are evident and separated into $N M 1$ and $N M 2$. NM1 responses occur when the encroaching stimulus is the same color as the main receptive field stimulus; thus, neither match the conditional cue. NM2 responses occur when the encroaching stimulus is the opposite color from the main stimulus and thus matches the conditional cue.

the CD paradigm had no detrimental effect on the response elicited by the single stimulus in the receptive ficld until a second array stimulus encroached upon the classical receptive field. The usual result of entry into the receptive field by a second stimulus was a greatly diminished difference between $M$ and NM conditions, with both responses attaining a more moderate level of sustained activation. In general, all stimuli used were at least moderately effective when presented by themselves in the receptive field. Because of the relatively large receptive fields of V4 neurons, and the initial centering of one stimulus in the receptive field, increasing the number of stimuli in the array usually resulted in a symmetrical encroachment into the field from both sides. Figure 8 shows one case of a small field that was bracketed by stimuli in a slightly asymmetric manner. The cartoon on the left in Figure 8 depicts the inner margin of the receptive field contour line marking a $50 \%$ reduction in sensitivity from the field center. The locations of stimuli in the arrays used are marked by stimulus bars. Just one orientation is used in the cartoon, whereas the flanking stimuli are randomly permuted during the experiment. The receptive field stimulus (more or less centered in the field) is located to the lower left of the fixation target, at an eccentricity of about $4^{\circ}$ of visual angle. The responses of the neuron to the $M$ and NM conditions are shown in the rasters to the right for arrays of eight (upper) and 16 (lower) stimuli. Only responses to the primary stimulus (color, blue; orientation, $50^{\circ}$ ) are shown; the alternate color (red) was comparable in eliciting a response. With eight stimuli in the array (Fig. $8 A$ ) the responses to M and NM are clearly differ- entiated. Arrays of 16 stimuli, however, result in the flanking stimulus on the right being positioned in the central part of the receptive field, as indicated by the $50 \%$ line in Figure 8 . Nevertheless, when the primary receptive field stimulus matches the conditional cue (Fig. 8B, M) the response remains intact. However, in the NM condition two different responses can be distinguished and are separated in Figure $8 B$ into NM1 and NM2. In NM1 the encroaching stimulus was an NM stimulus as was the receptive field stimulus, and the elicited response was similar to other NM conditions. In NM2 the encroaching stimulus was the optimally oriented stimulus of the opposite color (in this case, red) and therefore the second stimulus matched the conditional cue. This stimulus combination produced a maintained response, presumably because there was in fact an optimally oriented color matching stimulus (the red one) in the receptive field. Given these results it seems reasonable that a mixture of several stimuli in the receptive field may produce overall intermediate activations similar to those observed in the majority of the neurons tested with larger array sizes.

A different observation was made in three of the 24 neurons; the most striking example is shown in Figure 9. In this case, increasing the array from four to eight stimuli resulted in the placement of an array stimulus into an apparent inhibitory zone. When the encroaching stimulus entered the inhibitory zone, the maintained $M$ response was suppressed or occluded. The NM response, however, is not affected in the same manner and appears to be, if anything, more evenly activated. This latter observation suggests the possibility of multiple mechanisms in the 
control of the feature-selection effect. A permuted selection of array stimuli was used in all of these experiments so that the stimulus in the suppressive zone was varied from trial to trial. This particular neuron, however, was not orientation sensitive and the color pairs were equally effective in driving the neuron. Excitatory or inhibitory subfields were not observed in these studies and V4 classical receptive fields are generally reported as homogeneous (Desimone and Schein, 1987). Therefore, the observed inhibitory zones are interpreted as being part of the suppressive surround. As expected, the engagement of these surrounds clearly alters the response patterns seen in the CD paradigm.

\section{Discussion}

The results of the present studies indicate that the feature-selective processing that occurs in extrastriate area V4 can be sustained by the memory of the relevant cue. The observed feature-selective differences in neuronal activity therefore cannot simply reflect a differential processing of the physical features of the visual scene as they exist but reveal an activc control process based on an implied goal. Neurons in several areas in inferior temporal cortex have been shown to maintain their discharge activity during delay periods in visual memory tasks (Fuster and Jervey, 1982; Miyashita and Chang, 1988). The discharge of such neurons, especially those in more anterior inferior temporal areas, was selective for particular sets of stimulus features that the monkey had to remember during such delays. The presence of clear anatomical connections between V4 and inferior temporal cortex suggests one avenue by which mnemonic representations of the cue information can gain access to V4.

The present studies further show that even after the selective processing has been established, the control remains dynamic and can be switched from one feature to another during the course of a behavioral trial. Changing the selected feature results in a dramatic change in area V4. Neurons with the ncwly selected feature in their receptive fields suddenly become activated without a concomitant change in the stimulus in their classical receptive fields. Across the topographic map of V4 the activity of the newly selected targets pops out, whereas the deselected targets fade to the background levels of all other background objects. The time required to switch selection from one feature to another is appreciably longer than the amount of time it takes for visual information to arrive initially in area V4.

Fischer and Boch $(1981,1985)$ reported a reactivation of neural activity in a suppressed saccadic eye movement task when the fixation target disappeared and the monkeys were free to make an eye movement to a peripheral target in the neuron's receptive field. Because in their paradigms the reactivation was associated with the release of the fixation requirement at the central target, and could not be conclusively demonstrated in a peripheral attention task, they concluded the reactivation was due to a change in visual sensitivity related to the active state of fixation. While these observations are similar to the pop-out changes seen in the CS paradigm, they are also compatible with the Fischer and Boch (1985) proposal in that they represent a shift in directed focal attention. One clear difference between those studies and the present one is their report that the reactivation activity faded with prolonged training of 3-5 weeks. The monkeys in the present experiments were trained for 4-6 months before recording began.

Are the feature-selective effects observed in the present study

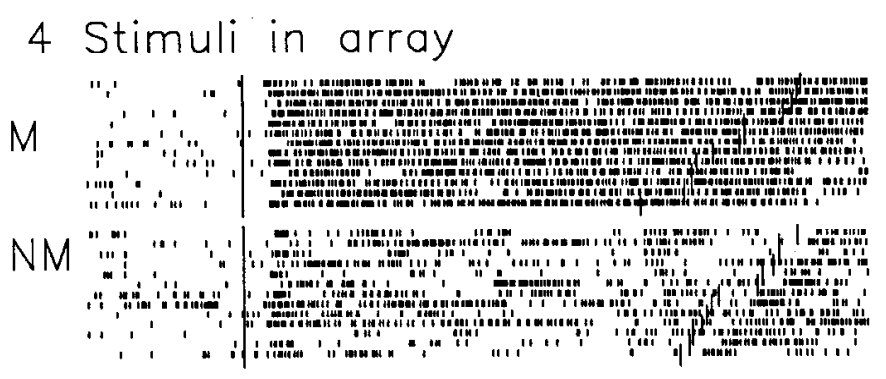

8 Stimuli in array

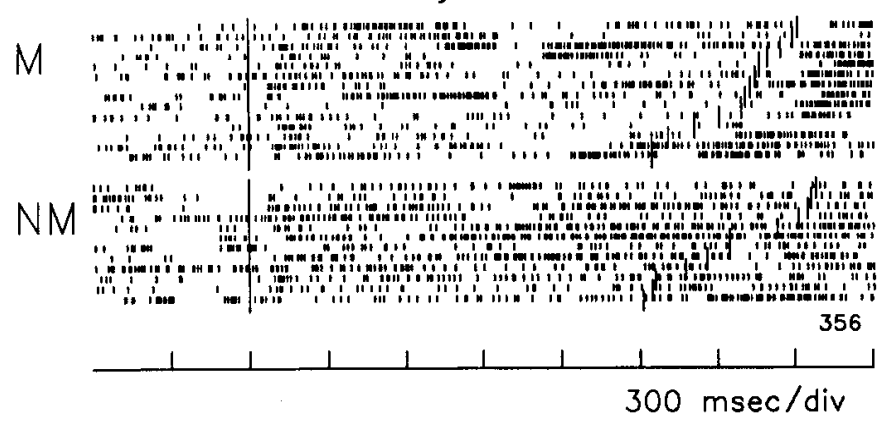

Figure 9. Entry of array stimuli into suppressive zones: discharge responses of a V4 neuron to $\mathrm{M}$ and NM conditions in the standard $\mathrm{CD}$ paradigm for arrays containing four and eight stimuli. In the upper raster pair the responses are clearly differentiated for matching $(M)$ and nonmatching $(N M)$ conditions. Increasing the number of stimuli in the array to eight apparently placed one (or two) stimuli into a suppressive surround with the resulting clear effect of shutting off the response to the $M$ condition.

based on feedforward control systems that separate or group objects on the basis of elemental differences in physical properties? For example, simple texture segmentation depends on a bottom-up processing built on the evaluation of local differences in luminance contrast or orientation contrast and is sensitively dependent upon the texture gradient (Nothdurft, 1985; Landy and Bergen, 1991). The properties of feedforward systems may in fact explain the rapid, effortless, preattentive discriminations of texture areas having suitable differences in luminance or orientation contrast. Such systems appear to be the physiological basis for the control of texture segmentation based on luminance (Nothdurft and $\mathrm{Li}, 1985$ ) or orientation differences (Nothdurft, 1991; Knierim and Van Essen, 1992). Some of the properties of feature selection observed in the present studies, for example, cue-dependent differential activation and continued differentiation in the absence of a cue stimulus, are not readily compatible with feedforward explanations. The observed properties suggest a feedback control system.

Many of the V4 neurons studied responded to a reasonably wide band of colors and luminances. In the conditional paradigms there were no clear limits; essentially any mixture, including the two best "colors," could be paired and differential driving could be obtained. This differential activation implies that the control system is able to shut down effectively the synaptic impact of all but one of many "color" inputs. The difficulty in organizing a feedback system with such specificity, particularly one that may be using a memory or a nonvisual source (I Iaenny et al., 1988), suggests that what is bcing sclcctcd may not be a particular color but instead a particular relationship of the selected color to other color features in general. Essentially this is a restatement of the observation that the pat- 
tern of activity during the $C D$ paradigm segments the scene into two maps, potential targets and background objects. The basis for segmentation may be color, as it would seem to be in this case, or alternatively it may be that the color values or differences are themselves simply a reflection of a more generalized comparator system, such as suggested by the lesion study of Schiller and Lee (1991) or the psychophysical studies of Treisman and Gormican (1988). A comparator role for V4 neurons, comparing activity in the classical receptive field with that in the suppressive surround, has already been proposed as a means to solve color constancy problems (Schein and Desimone, 1990). Extension of these ideas to a larger arena, including form (Desimone and Schein, 1987) and motion (Allman et al., 1985) but retaining the basic comparison between the classical receptive field versus peripheral surround, might provide a mechanism whereupon feedback could then select a particular relationship or ratio of activity.

The term "pop-out" is used in this article to describe the parallel emergence of the neural representation of a set of stimuli sharing a particular feature. The term is used to emphasize the similarities between the parallel processing described here for neural processes and those posed in the psychophysical descriptions of visual behavior under similar conditions. In psychophysical contexts the pop-out phenomenon is usually described in association with the capturing of attention by an object with a particularly distinctive or unique feature. The detection process for such an object appears to work in parallel across a scene or set of objects. Objects that have no particularly conspicuous feature or are distinct only by particular combinations of features require a slower process for detection that appears to use a serial examination of each object. Wolfe et al. (1989) suggested that these two processes work together with the parallel process identifying a limited set of objects that serial process can then examine. The parallel process in this scenario is a potential match with the feature-selective process described in this article. If these processes are related, however, the neural data indicate a major role for attentional control in early parallel visual processes that is contrary to current psychophysical models.

Are there interactions between stimuli in the suppressive surround and the classical receptive field in the CD paradigm? While it was easy to confirm the rich set of interactions for length and width selectivity within the classical receptive field reported by Desimone and Schein (1987), confirmation of the suppressive surrounds was surprisingly more difficult. In the anesthetized, paralyzed animal the surround fields in area V4 are bilateral and quite large, extending $15^{\circ}$ or more from the classical receptive field (Schein and Desimone, 1990; Desimone et al., 1993). On the basis of size alone, most if not all of the array stimuli in the $C D$ paradigm should have been located within the boundaries of the surround field. Yet using the simple discrimination paradigm there were only a few cases where multiple stimuli produced a suppressive effect relative to single stimuli, and then it was from sites immediately adjacent to the classical field. In the $C D$ task increasing the number of stimuli in the array did not appear to produce changes until the additional stimuli encroached upon the classical receptive field. During the $C D$ task the activity was generally greater in the $M$ condition and less in NM condition than the activity observed in response to single stimuli. The influence of the surround would therefore seem to be something other than a simple center-surround weighting. Other possibilities are that the surround stimuli were too small relative to the size of the surround or that the mixture of multiple stimuli within the surround canceled out the suppressive effects. It is also possible that the surround interactions in V4 are different in the awake behaving animal.

What is occurring during the presentation of the array in the CD paradigm, and is the observed feature-sclcctive process a focal attentive process? One possibility is that during this period the monkey is scanning the different stimuli in the array with a focal attentive mechanism while fixating the central spot. However, the discharge activity for the $\mathrm{M}$ condition maintains a constant level of activity without any major interruptions, as would be expected if the process resulted from a serial scan of the different stimuli. The consistent differences in the initial responses of many neurons to the matching conditions are also not compatible with a probabilistic selection of the one stimulus in the array that happens to be in the receptive field on each trial. The CS paradigm illustrates that selection changes can occur during the trial, but the changes require a considerable amount of time to occur, about an order of magnitude longer than the focal attentive scanning times of psychophysical experiments for such simple color feature differences (Treisman and Gelade, 1980; Krose and Julesz, 1989). The featurc-sclcctive process appears to be a relatively slow, parallel process that is not automatic in the sense of the preattentive processes discussed above in relation to texture discriminations. The result of this process in V4 is an activity biased map of the potential targets for the conditional task. These conditions bear a striking resemblance to the "saliency map" posed by Koch and Ullman (1985). In their model of attentive processes the saliency map brought together information from several feature maps to produce a single map of conspicuous items. A spatially restricted selection process was then used to read out information from the saliency map to a central representation. The spatially restrictive, focal attentive process reported by Moran and Desimone (1985) in V4 offers a second interesting parallel to the model. However, whether the feature-selective processes in V4 can be extended to features other than color and luminance remains to be demonstrated.

The feature-selective processes described in these experiments indicate that in area V4 visual processing attains a level beyond the basic description of the physical properties of the visual scene. The CD paradigm appears to provide a window onto the processes that precede focal attention and highlight specific stimuli for further consideration at various locations. The featureselective process can dynamically change the V4 topographic map of activity from moment to moment, causing the representations of stimuli to pop out or fade away as the relevance of different stimulus features change. Furthermore, the differentiation of stimuli can be based on the memory of relevant cues. Clearly what has begun to emerge is a view that visual processing within V4 is concerned not just with an object's stimulus features, but also with selective processes that weigh the similarities and differences between objects and the goals of visual search.

\section{References}

Allman J, Miezin F, McGuinness E (1985) Stimulus specific responses from beyond the classical receptive field: neurophysiological mechanisms for local-global comparisons in visual neurons. Annu Rev Neurosci 8:407-430.

Desimone R, Schein S (1987) Visual properties of neurons in area V4 of the macaque: sensitivity to stimulus form. J Neurophysiol 57:835868 . 
Desimone R, Moran J, Schein SJ, Mishkin M (1993) A role for the corpus callosum in visual area V4 of the macaque. Vis Neurosci 10 : 159-171.

Fischer B, Boch R (1981) Selection of visual targets activates prelunate cortical cells in trained rhesus monkey. Exp Brain Res 41:431-433.

Fischer B, Boch R (1985) Peripheral attention versus central fixation modulation of the visual activity of prelunate cortical cells of the rhesus monkey. Exp Brain Res 345:111-123.

Fuster JM, Jervey JP (1982) Neuronal firing in the inferotemporal cortex of the monkey in a visual memory task. J Neurosci $2: 361-$ 375.

Gattass R, Sousa APB, Gross CG (1988) Visuotopic organization and extent of V3 and V4 of the macaque..J Neurosci 8:1831-1845.

Haenny PE, Schiller PH (1988) State dependent activity in monkey visual cortex. I. Single cell activity in V1 and V4 on visual tasks. Exp Brain Res 69:225-244.

Haenny PE, Maunsell JHR, Schiller PH (1988) State dependent activity in monkey visual cortex. II. Retinal and extraretinal factors in V4. Exp Brain Res 69:245-259.

Knierim JJ, Van Essen DC (1992) Neuronal responses to static texture patterns in area V1 of the alert macaque monkey. J Neurophysiol 67: $961-980$.

Koch C, Ullman S (1985) Shifts in selective visual attention: towards the underlying neural circuitry. Hum Neurobiol 4:219-227.

Krose BJA, Julesz B (1989) The control and speed of shifts of attention. Vision Res 29:1607-1619.

Landy MS, Bergen JR (1991) Texture segregation and orientation gradient. Vision Res 31:679-691.

Maunsell JHR, Sclar G, Nealey TA, DePriest DD (1991) Extraretinal representations in area V4 in the macaque monkey. Vis Neurosci 7:561-573.

Miyashita Y, Chang HS (1988) Neuronal correlate of pictorial shortterm memory in the primate temporal cortex. Nature 331:68-70.
Moran J, Desimone R (1985) Selective attention gates visual processing in the extrastriate cortex. Science 229:782-784.

Motter BC (1993) Focal attention produces spatially selective processing in visual cortical areas $\mathrm{V} 1, \mathrm{~V} 2$ and $\mathrm{V} 4$ in the presence of competing stimuli. J Neurophysiol 70:909-919.

Motter BC (1994) Neural correlates of attentive selection for color or luminance in extrastriate area V4. J Neurosci 14:2178-2189.

Motter BC, Poggio GF (1984) Binocular fixation in the rhesus monkey: spatial and temporal characteristics. Exp Brain Res 54:304-314.

Nothdurft HC (1985) Sensitivity for structure gradient in texture discrimination tasks. Vision Res 25:1957-1968.

Nothdurft HC (1991) Texture segmentation and pop-out from orientation contrast. Vision Res 31:1073-1078.

Nothdurft HC, Li CY (1985) Texture discrimination: representation of orientation and luminance differences in cells of the cat striate cortex. Vision Res 25:99-113.

Schein SJ, Desimone R (1990) Spectral properties of V4 neurons in the macaque. J Neurosci 10:3369-3389.

Schiller PH, Lee K (1991) The role of the primate extrastriate area $\mathrm{V} 4$ in vision. Science 251:1251-1253.

Spitzer H, Desimone R, Moran J (1988) Increased attention enhances both behavioral and neuronal performance. Science 240:338-340.

Treisman A, Gelade G (1980) A feature integration theory of attention. Cognit Psychol 12:97-136.

Treisman A, Gormican S (1988) Feature analysis in early vision: evidence form search asymmetries. Psychol Rev 95:15-48.

Wolfe JM, Cave KR, Franzel SL (1989) Guided search: an alternative to the feature integration model for visual search.J Exp Psychol [Hum Percept] 15:419-433.

Wurtz RH (1969) Visual receptive fields of striate cortex neurons in awake monkeys. J Neurophysiol 32:727-742. 